\title{
Perfil dos óbitos fetais em gestantes adolescentes no Acre no período de 2014 a 2016
}

\author{
Profile of fetal deaths in pregnant adolescents in \\ Acre (Brazil) in the period from 2014 to 2016
}

\section{Ruth Silva Lima da Costa' (1) Joéden Zegarra da Silva ${ }^{2}$ (1) Eduarda Monteiro da Silva Kagy 3 (1) Emeli do Nascimento Nery ${ }^{4}$ (1) Eder Ferreira de Arruda 5 (1)}

\footnotetext{
1'Autora para correspondência. Centro Universitário UNINORTE (Rio Branco). Acre, Brasil. ruttylyma@gmail.com ${ }^{2-5}$ Centro Universitário UNINORTE (Rio Branco). Acre, Brasil. joeden.zegarra174@gmail.com, duda.kagy@gmail.com, emelimds@gmail.com, ederarrud@gmail.com
}

RESUMO | OBJETIVO: Analisar o perfil de óbitos fetais em gestantes adolescentes no Acre no período de 2014 a 2016. MÉTODO: Estudo transversal, retrospectivo e exploratório. A população do estudo foi composta por todos os casos de óbitos fetais ocorridos em adolescentes em Rio Branco - Acre e registrados entre 2014 a 2016 disponíveis no Departamento de Informática do Sistema Único de Saúde (DATASUS). RESULTADOS: Ocorreram 85 óbitos fetais, predominando: mães na faixa etária de 15 a 19 anos 78 (92,0\%), com 4 a 7 anos de estudo $38(44,7 \%)$ e duração da gestação de 28 a 31 semanas 20 (23,5\%), correspondendo ao $7^{\circ}$ mês de gravidez. A maioria das gestações era de fetos únicos $83(97,6 \%)$, do sexo feminino $48(57,0 \%)$, com peso fetal variando entre $1500 \mathrm{~g} \mathrm{a}$ 2499g $23(27,1 \%)$, que foram a óbito antes do momento do parto $79(93,0 \%)$, tendo como a principal causa de morte algumas afecções originadas no período perinatal 78 (92,0\%). CONCLUSÃO: A maioria dos óbitos fetais ocorreu entre a $28^{\mathrm{a}}$ e $36^{\mathrm{a}}$ semana, em mães na faixa etária de 15 a 19 anos, com baixa escolaridade, mostrando assim a necessidade de estratégias para redução da gravidez na adolescência e aumento da cobertura de pré-natal.

DESCRITORES: Óbito Fetal. Mortalidade perinatal. Morte prematura. Gravidez na adolescência.

\begin{abstract}
OBJECTIVE: To analyze the profile of fetal deaths in pregnant women in Acre from 2014 to 2016. METHOD: Cross-sectional, retrospective and exploratory study. The study population consisted of all cases of fetal deaths occurred in adolescents in Rio Branco - Acre and registered between 2014 and 2016 available at the Department of Informatics of the Unified Health System (DATASUS). RESULTS: There were 85 fetal deaths, predominantly: mothers aged 15-19 years $78(92.0 \%)$, with $4-7$ years of study $38(44.7 \%)$ and gestation duration 28 to 31 weeks 20 (23.5\%), corresponding to the 7th month of pregnancy. Most pregnancies were single fetuses $83(97.6 \%)$, female $48(57.0 \%)$, with fetal weight ranging from $1500 \mathrm{~g}$ to $2499 \mathrm{~g} 23$ (27.1\%), who died before the time. $79(93.0 \%)$, and the main cause of death was some diseases originating in the perinatal period 78 (92.0\%). CONCLUSION: Most fetal deaths occurred between the 28th and 36th week in mothers aged 15-19 years, with low education, thus showing the need for strategies to reduce teenage pregnancy and increase prenatal coverage.
\end{abstract}

DESCRIPTORS: Fetal death. Perinatal mortality. Premature death. Teenage pregnancy. 


\section{Introdução}

A adolescência é a fase da vida compreendida entre a infância e a fase adulta, caracterizada pelo processo de crescimento, desenvolvimento e amadurecimento ${ }^{1}$.

A Organização Mundial de Saúde (OMS) define adolescência como sendo o período da vida que começa aos 10 anos e termina aos 19 anos completos. Para a OMS, a adolescência é dividida em três fases: préadolescência que vai dos 10 aos 14 anos, a adolescência compreendida entre os 15 aos 19 anos completos e a Juventude que varia dos 15 aos 24 anos $^{2}$. No entanto, de acordo com o Estatuto da Criança e do Adolescente (ECA), a adolescência envolve a faixa etária entre 12 a 18 anos $^{3}$.

Nessa etapa da vida, os adolescentes podem iniciar a atividade sexual sem a devida utilização dos métodos contraceptivos, não somente pela falta de conhecimento da forma de utilização dos mesmos, mas também pelo sentimento de invulnerabilidade muito comum nessa fase 4 o que pode levar a riscos de contrair infecções sexualmente transmissíveis (IST's), e ocorrer uma gravidez indesejada ${ }^{5}$.

Frente a isso, a literatura tem evidenciado que as mães adolescentes apresentaram maior proporção de partos prematuros, recém-nascidos com baixo peso ao nascer e maior número de partos de natimortos ${ }^{6}$.

Define-se óbito fetal, morte fetal, nascido morto/natimorto como a morte de um produto da concepção, antes da expulsão ou da extração completa do corpo da mãe, independente da duração da gravidez ${ }^{7}$. No Brasil, determina-se como óbito fetal o feto com peso ao nascer igual ou inferior a 500 gramas, idade gestacional de 22 semanas (154 dias) e/ou conceptos com comprimento corpóreo de 25 centímetros cabeçacalcanhar ou mais e confirma-se o óbito após o feto não ter respiração, batimentos cardíacos, pulsação do cordão umbilical e não expressar nenhum tônus muscular ${ }^{\text {. }}$.

A mortalidade fetal é um sério problema da saúde pública, porém tem sido dada pouca importância às causas desses óbitos, visto que, sua ocorrência pode se associar a uma eventualidade fatal, apesar dessas mortes serem influenciadas pelas mesmas circunstâncias e etiologias que a mortalidade neonatal precoce ${ }^{9}$.
Devido ao fato de não se tratar de um indicador de saúde, esses óbitos vêm sendo negligenciados de longas datas pelos serviços de saúde, não sendo a temática incorporada nas discussões e nas análises sobre sua ocorrência e tampouco são destinados investimentos específicos para redução da natimortalidade ${ }^{10}$.

Nesse contexto, o presente artigo tem o objetivo analisar o perfil de óbitos fetais em gestantes adolescentes no Acre no período de 2014 a 2016.

\section{Método}

Trata-se de um estudo transversal, retrospectivo, exploratório, com coleta de dados secundários obtidos no Departamento de Informática do Sistema Único de Saúde (DATASUS), tabulados a partir do TABNET utilizando os dados de Estatísticas VitaisMortalidade e Nascidos vivos, através dos seguintes passos: DATASUS - Informações em Saúde (TABNET) Estatísticas Vitais - Mortalidade e Nascidos Vivos Mortalidade - 1996 a 2017, pela CID-10. Para a coleta de dados foram selecionadas as seguintes variáveis: idade da mãe, grau de escolaridade, duração da gestação, tipo de gravidez, peso do feto ao nascer, sexo do feto, investigação do óbito e as causas dos óbitos.

A amostra foi composta por 85 óbitos casos de óbitos fetais provenientes de mães adolescentes de 10 a 19 anos, na cidade de Rio Branco no período de janeiro de 2014 a dezembro de 2016. Foram excluídos os dados de óbitos que não apresentavam as informações referentes às variáveis de estudo.

Os dados foram apresentados por meio da estatística descritiva em frequência absoluta e percentual, e organizados em tabelas de acordo com as variáveis analisadas. Para produção dos gráficos foi utilizada a ferramenta do Microsoft Office Excel 2010.

O trabalho não foi submetido ao Comitê de Ética em Pesquisa - CEP local, por tratar-se de estudo em fontes secundárias de domínio público e não se enquadrar dentro da legislação do Conselho Nacional de Ética e Pesquisa/ Ministério da Saúde (CONEP/MS), Resolução de 466/2012. 


\section{Resultados}

A tabela 01 evidencia os dados sócio demográficos das mães de 10 a 19 anos cujos conceptos nasceram natimortos na cidade de Rio Branco-Acre no período de estudo. Observa-se que a maioria das adolescentes encontrava-se na faixa etária de 15 a 19 anos com 78 (92,0\%). Referente aos anos de estudos, elas haviam estudado, em sua maioria, de 4 a 7 anos com 38(44,7\%), correspondendo ao ensino fundamental.

Tabela 1. Perfil Sócio demográfico de mães adolescentes de conceptos natimortos em Rio Branco - Acre no período de 2014 a 2016

\begin{tabular}{lcc}
\hline Variável & N & \% \\
\hline Faixa etária & 7 & 8,2 \\
10 a 14 anos & 78 & 92,0 \\
15 a 19 anos & & 10,6 \\
Anos de Estudo & 9 & 44,7 \\
1 a 3 anos & 38 & 23,5 \\
4 a 7 anos & 20 & 2,4 \\
12 ou mais 11 anos & 2 & 18,8 \\
Ignorado & 16 & 2 \\
\hline
\end{tabular}

Fonte: TABNET-DATASUS, 2018

Concernente aos dados expostos na Tabela 2, referente à duração da gestação, observa-se a predominância de óbitos fetais ocorridos entre $22^{\mathrm{a}}$ a $27^{\mathrm{a}}$ semanas (correspondente $7^{\circ}$ mês de gravidez) e entre a $28^{\mathrm{a}}$ a $31^{\mathrm{a}}$ semanas (correspondendo ao $8^{\circ}$ mês gestacional), representados pela frequência de $20(23,5 \%)$ cada. Quanto ao tipo de gravidez a maioria dos casos era de gestação de feto único 83 (97,6\%).

Tabela 2. Dados da Gestação de mães adolescentes de conceptos natimortos em Rio Branco - Acre no período de 2014 a 2016

\begin{tabular}{lcc}
\hline Variável & $N$ & $\%$ \\
\hline Duração da Gestação & 6 & 7,1 \\
Menos de 22 semanas & 18 & 21,2 \\
22 a 27 semanas & 20 & 23,5 \\
28 a 31 semanas & 20 & 23,5 \\
32 a 36 semanas & 15 & 17,6 \\
37 a 41 semanas & 6 & 7,1 \\
Ignorado & & 97,6 \\
Tipo de Gravidez & 83 & 1,2 \\
Única & 1 & 1,2 \\
Dupla & 1 & \\
Tripla & & \\
\hline
\end{tabular}

Fonte: TABNET-DATASUS, 2018 
Relativo aos dados dos natimortos quanto ao peso fetal, a maioria deles nasceu com o peso entre $1500 \mathrm{~g}$ a $2499 \mathrm{~g}$ $23(27,1 \%)$ e entre 500g a 999g 20 (23,5\%), sendo que no tocante ao momento da ocorrência do óbito, a maior parte ocorreu antes do parto 79 (93,0\%). Quanto ao sexo do concepto, o maior número era do sexo feminino com $48(57,0)$ e no que se refere a investigação dos óbitos $80(94,0 \%)$ foram investigados.

Tabela 3. Dados dos natimortos de mães adolescentes em Rio Branco - Acre no período de 2014 a 2016

\begin{tabular}{|c|c|c|}
\hline Variável & $\mathrm{N}$ & $\%$ \\
\hline \multicolumn{3}{|l|}{ Peso Fetal ao nascer } \\
\hline 500g a $999 g$ & 20 & 23,5 \\
\hline $1000 \mathrm{~g}$ a $1499 \mathrm{~g}$ & 17 & 20,0 \\
\hline $1500 \mathrm{~g}$ a $2499 \mathrm{~g}$ & 23 & 27,1 \\
\hline $2500 \mathrm{~g}$ a $2999 \mathrm{~g}$ & 8 & 9,4 \\
\hline 3000g a 3999g & 13 & 15,3 \\
\hline $4000 \mathrm{~g}$ ou mais & 1 & 1,2 \\
\hline Ignorado & 3 & 3,5 \\
\hline \multicolumn{3}{|l|}{ Momento do Óbito } \\
\hline Antes do Parto & 79 & 93,0 \\
\hline Durante o parto & 5 & 6,0 \\
\hline Ignorado & 1 & 1,0 \\
\hline \multicolumn{3}{|l|}{ Sexo do Concepto } \\
\hline Feminino & 48 & 57,0 \\
\hline Masculino & 36 & 42,0 \\
\hline Ignorado & 1 & 1,0 \\
\hline \multicolumn{3}{|l|}{ Investigação do óbito } \\
\hline Sim & 80 & 94,0 \\
\hline Não & 5 & 56,0 \\
\hline
\end{tabular}

Fonte: TABNET-DATASUS, 2018

Os dados evidenciados na tabela 04, demostram que a grande maioria dos óbitos de natimortos foi por algumas afecções originadas no período perinatal 78 (92,0\%). 


\begin{tabular}{lcc}
\hline Variável & N & $\%$ \\
\hline Principais causas dos óbitos & 3 & $(3,0 \%)$ \\
Algumas Doenças Infecciosas e Parasitárias & 78 & $(92,0 \%)$ \\
Algumas Afecções Originadas no Período Perinatal & 4 & $(5,0 \%)$ \\
Malformações Congênitas e Anomalias Cromossômicas & & \\
\hline Fonte: TABNET-DATASUS, 2018 &
\end{tabular}

\section{Discussão}

Em conformidade com os dados sóciodemográficos de mães adolescentes de conceptos natimortos encontrados nesse estudo, a pesquisa realizada por Bulhões e colaboradores (2018) ${ }^{11}$ que retratou a prevalência de recém-nascidos pré-termo de mães adolescentes, confirmou que a gravidez em gestantes adolescentes favorece o parto prematuro o que contribui para evolução do óbito fetal. Observou-se ainda no estudo que de 2.357 mulheres grávidas estudadas, 4\% eram menores de 18 anos e esse grupo foi o que apresentou mais complicações (dentre eles o óbito fetal), corroborando com os nossos achados, uma vez que a maioria das gestantes encontrava-se na faixa etária de 15 a 19 anos.

Referente à escolaridade os dados encontrados estão de acordo com estudo realizado no Acre em 2018 sobre a ocorrência de partos prematuros em adolescentes que também evidenciou a baixa escolaridade entre as adolescentes gestantes, pois $47 \%$ estudaram de 4 a 7 anos $^{12}$.

Um estudo que comparou a atenção ao pré-natal e os fatores de risco associados à prematuridade e baixo peso ao nascer em capital do nordeste brasileiro, evidenciou que a baixa escolaridade materna está diretamente relacionada ao aumento de chances para a ocorrência de prematuridade e baixo peso ao nascer ${ }^{13}$.

Segundo a OMS e a Assembleia Mundial da Saúde, a idade gestacional ideal mínima para o parto é de 37 semanas, o bebê que nascer antes desse prazo é considerado prematuro, podendo ter mais chances de desenvolver complicações durante e após seu nascimento, incluindo o óbito fetal ${ }^{14}$.
O estudo de Crizóstomo e colaboradores $(2016)^{15}$ sobre o perfil do óbito de natimortos com enfoque nos determinantes materno encontrou um resultado semelhante ao encontrado nesse estudo, onde a maioria dos partos de natimortos ocorreu com menos de 32 semanas de gestação $(48,6 \%)$.

A mortalidade fetal ou natimortalidade se divide em precoce, intermediária e tardia. A precoce refere-se e aos abortos e está compreendida no período entre a concepção e a vigésima semana de gestação, no qual o feto tem um peso aproximado de quinhentos gramas (500g). A morte fetal intermediária ocorre entre a $20^{\mathrm{a}}$ e a $28^{\mathrm{a}}$ semana de gestação (com pesos fetais entre 500 e $1000 \mathrm{~g})$ e a fetal tardia entre a $28^{\mathrm{a}}(1000 \mathrm{~g}$ ou mais) e o parto ${ }^{16}$. O resultado encontrado no presente estudo evidenciou que no período avaliado a maioria dos óbitos ocorreu ente a $28^{\mathrm{a}}$ e $36^{\mathrm{a}}$ semana, correspondendo ao período de morte fetal tardia.

Os achados de Lansky (2013), retratam que o elevado número de natimortos no final de uma gestação demostra uma estreita relação entre esses óbitos e a qualidade da saúde serviços que prestam cuidados a mulheres grávidas?.

No estudo de Silva et al. (2016) ${ }^{17}$, uma das conclusões de fatores de risco para a ocorrência da natimortalidade é o baixo peso ao nascer, corroborando também com os nossos achados. No que tange aos achados de Assis (2013) ${ }^{18}$, onde de um total de 71 óbitos, para os fetos $>2.500$ gramas a frequência de natimortos foi de $30 \%(n=22)$, entretanto a maioria dos óbitos concentrou-se nos de baixo peso $(<2.500 \mathrm{~g})$ onde a frequência foi de $63 \%(n=45)$. Isso deve-se porque quanto menor o peso do feto menos viabilidade de sobrevivência ele tem, confirmando assim os achados encontrados na presente pesquisa. 
No que diz respeito ao momento da ocorrência do óbito, os dados do presente estudo corroboram com o estudo de Crizóstomo e colaboradores (2016) ${ }^{15}$, onde a maioria dos óbitos também ocorreu antes do trabalho de parto $(87,7 \%)$ e apresentando peso ao nascer menor que 1500 gramas $(39,1 \%)$.

Estudos têm evidenciando que no Brasil a maioria dos óbitos fetais ocorrem no período do pré-parto ${ }^{19-20}$ sugerindo a existência de fragilidades na atenção ao pré-natal e correlacionando os fatores associados que podem levar ao óbito do concepto, como a idade materna, a baixa renda e a baixa escolaridade.

Quanto a natimortalidade, quando comparada ao sexo do concepto, os nossos achados divergem do estudo de Silva et al. (2016) ${ }^{21}$ que demostrou em seu estudo que dos óbitos fetais, a maioria pertencia ao sexo masculino, com peso entre 500 e 1499 gramas e idade gestacional de 28 a 36 semanas e também dos achados de Chiavegatto, Filho e Laurenti (2012) ${ }^{22}$, onde os óbitos fetais também ocorreram predominantemente no sexo masculino.

Referente à investigação dos óbitos nossos achados corroboram com o estudo de Pereira et al. (2019) ${ }^{23}$ que evidenciou que dos 5.318 óbitos que ocorreram, a maioria também foram investigados. Esse fato é considerado um avanço, pois de acordo com recomendações do Ministério da Saúde a partir de 2010, houve a obrigatoriedade da vigilância dos óbitos materno, infantil e fetal pelos profissionais envolvidos na atenção materno-infantil/8.

Com relação às principais causas dos óbitos, nossos achados corroboram com os de Pereira et al. (2016) ${ }^{24}$ que apontou que as afecções no período perinatal estiveram associadas ao grupo de causas de morte com maior percentual $(87,5 \%)$ e $77,4 \%$ das mortes que foram classificadas como evitáveis, ressaltando que os óbitos decorrentes de causas evitáveis põem em questão a qualidade da atenção pré-natal, obstétrica e neonatal.

Este estudo apresentou algumas limitações que merecem ser mencionadas, no que se refere ao o uso de dados secundários, que não permitem ao pesquisador controlar possíveis erros decorrentes de digitação e de registro, além de possíveis subnotificações. Apesar disto, acredita-se que, por se tratar de dados nacionais oficiais e de preenchimento obrigatório no serviço de saúde, os dados coletados permitiram o alcance dos objetivos propostos no presente estudo.

\section{Conclusão}

Neste estudo, identificou-se que a maioria dos óbitos fetais ocorridos no período compreendeu a faixa etária de 15 a 19 anos, de mães com baixa escolaridade, onde a morte fetal ocorreu entre a $28^{\mathrm{a}}$ e $36^{\mathrm{a}}$ semana, correspondendo ao período de morte fetal tardia. A maioria das gestações eram únicas, de conceptos de $1500 \mathrm{~g}$ a $2499 \mathrm{~g}$, sexo feminino, que foram a óbito antes do parto, cujos óbitos foram investigados e as principais causas foram definidas a partir do CID 10 como algumas afecções originadas no Período Perinatal.

Mediante a isso, evidencia-se que existe a necessidade da implantação de estratégias para a redução da mortalidade perinatal e dentre elas podemos destacar a redução da gravidez na adolescência, o aumento da cobertura pré-natal e o fortalecimento da vigilância dos óbitos fetais e infantis, bem como a estruturação de comitês de prevenção do óbito, para o enfrentamento dessa problemática.

\section{Contribuições dos autores}

Silva JZ, Nery EN e Kagy EMS foram responsáveis pela coleta de dados, análise e interpretação dos resultados e escrita do artigo. Da Costa RSL analisou e interpretou os dados e supervisionou a pesquisa e produção do artigo.

\section{Conflitos de interesses}

Nenhum conflito financeiro, legal ou político envolvendo terceiros (governo, empresas e fundações privadas, etc.) foi declarado para nenhum aspecto do trabalho submetido (incluindo, mas não se limitando a subvenções e financiamentos, participação em conselho consultivo, desenho de estudo, preparação de manuscrito, análise estatística, etc.). 


\section{Referências}

1. Organização Pan-Americana da Saúde. Ministério da Saúde. Saúde e sexualidade de adolescentes. [Internet]. 2017. Disponível: http://bvsms.saude.gov.br/bvs/publicacoes/saude_sexualidade_ adolescente_construindo_equidade_sus.pdf

2. Ministério da Saúde. Secretaria de Atenção à Saúde. Área de Saúde do Adolescente e do Jovem. Marco legal: saúde, um direito de adolescentes. Brasília: Editora do Ministério da Saúde; 2007.

3. Ministério da Saúde. Estatuto da Criança e do Adolescente ECA. Ministério da Saúde. 3.ed. Brasília: Editora do Ministério da Saúde; 2008.

4. Ministério da Saúde. Secretaria de Atenção à Saúde. Departamento de Atenção Básica. Saúde sexual e saúde reprodutiva. Brasília: Ministério da Saúde; 2010.

5. Menegatti L, Oliveira RB, Gama IL. Complicações na Gravidez na Adolescência. Facider Revista Científica. 2014;06:17-31.

6. Azevedo W, Diniz M, Fonseca E, Azevedo L, Evangelista CB. Complicações da gravidez na adolescência: revisão sistemática da literatura. Einstein. 2015;13(4): 618-626. doi: 10.1590/S167945082015RW3127

7. Lima KJ, Chaves CS, Gomes EO, Lima MA, Candeira ECP, Teófilo FKS et al. Análise da situação em saúde: a mortalidade fetal na $10^{a}$ região de saúde do Ceará. Rev Bras Promoç Saúde. 2017;30(1):307. doi: $\underline{10.5020 / 18061230.2017}$

8. Brasil. Ministério da Saúde. Portaria $n^{\circ} 72$, de 11 de janeiro de 2010. Estabelece que a vigilância do óbito infantil e fetal é obrigatória nos serviços de saúde (públicos e privados) que integram o Sistema único de Saúde. Brasília: Ministério da Saúde; 2015.

9. Lansky S. Mortalidade fetal: mortes invisíveis e evitáveis. In: Bittencourt DAS, Dias MAB, Wakimoto MD, organizadores. Vigilância do óbito materno, infantil e fetal e atuação em comitês de mortalidade. Rio de Janeiro: EAD/ENSP; 2013. p.123-33.

10. Brasil. Manual de vigilância do óbito infantil e fetal e do comitê de prevenção do óbito infantil e fetal. 2.ed. Brasília: Ministério da Saúde; 2009.

11. Bulhões TRB, Alves JB, Moreno CA, Silva TB, Dutra LP. Prevalência de Recém Nascidos Pré-Termo de Mães Adolescentes. Id on Line Revista de Psicologia. 2018;11(39):84-96. doi: 10.14295/ idonline.v12i39.950

12. Hydall ARS, Duarte RN, Costa RSL. Partos Prematuros em Adolescentes em Rio Branco-Acre no ano de 2015. DêCiência em Foco. 2018;2(1):34-44.
13. Gonzaga ICA, Santos SLD, Silva ARV, Campelo V. Atenção pré-natal e fatores de risco associados à prematuridade $\mathrm{e}$ baixo peso ao nascer em capital do nordeste brasileiro. Ciência \& Saúde Coletiva. 2016;21(6):1965-1974. doi: 10.1590/141381232015216.06162015

14. Assembleia Mundial da Saúde; Organização Mundial da Saúde; DATASUS. Definições. [Internet]. 2018.[acesso em 25 de set. 2018]. Disponível em: http://www.datasus.gov.br/cid10/ V2008/WebHelp/definicoes.htm

15. Crizóstomo CD, Lira JAC, Ribeiro JF, Lima LC, Ferreira RSA, Vieira BMCS. Perfil do Óbito de Natimortos com enfoque nos determinantes maternos. Rev Pre Infec e Saúde. 2018;4:7152. doi: 10.26694/repis.v4i0.7152

16. Jacinto E, Aquino EML, Mota ELA. Mortalidade perinatal no município de Salvador, Bahia: evolução de 2000 a 2009. Rev de Saúde Pública. 2013;47(5):846-53. doi: 10.1590/S0034$\underline{8910.2013047004528}$

17. Mazotti BR, Zilly A, Ferreira H, Caldeira S, Silva RMM. Fatores epidemiológicos correlacionados ao risco para morte fetal: Revisão Integrativa da Literatura. Arq Ciênc Saúde. 2016;23(2):0915. doi: $10.17696 / 2318-3691.23 .2 .2016 .221$

18. Assis H, Siviero PCL, Drumond EF, Machado CJ. Óbitos fetais sob o prisma da evitabilidade: análise preliminar de um estudo para o município de Belo Horizonte. Cad Saúde Colet. 2014;22(3):314-7. doi: 10.1590/1414-462×201400030014

19. Vieira MSM, Siebert EC, Ceglio WQGW, Almeira MH, Batista TS, Freitas PF. Dificuldades para a identificação da causa do óbito fetal: como resolver? Rev Bras Ginecol Obstet. 2012;34(9):403-8. doi: $10.1590 / s 0100-72032012000900003$

20. Barbeiro FMS, Fonseca SC, Tauffer MG, Ferreira MSS, Silva FP, Ventura PM et al. Fetal deaths in Brazil: a systematic review. Rev Saúde Pública. 2015;49(22):1-15. doi: 10.1590/s0034$\underline{8910.2015049005568}$

21. Silva RMM, Mazotti BR, Zilly A, Ferreira H, Caldeira S. Análise da incidência de óbitos fetais entre municípios da nona regional de saúde do Paraná. Semina: Ciências Biológicas e da Saúde. 2016;37(1):33-42. doi: 10.5433/1679-0367.2016v37n1p33

22. Chiavegatto Filho ADP, Laurenti R. O sexo masculino vulnerável: razão de masculinidade entre os óbitos fetais brasileiros. Cad Saúde Pública. 2012; 28(4):720-728. doi: 10.1590/ s0102-311X2012000400011

23. Pereira SU, Lemos LM, Garcia E, Soares DB. Vigilância do óbito infantil no Espírito Santo, Brasil. Revi Bras de Pesquisa em Saúde/Brazilian Journal of Health Research. 2019;20(4):31-37. doi: $\underline{10.21722 / r b p s . v 20 i 4.24595}$

24. Pereira RC, Figueiroa MN, Barreto IC, Cabral LNC, Lemos MLC, Marques VLLR. Perfil epidemiológico sobre mortalidade perinatal e evitabilidade. Rev enferm UFPE on line. 2016;10(5):1763-72. doi: 10.5205/reuol.9003-78704-1-SM.1005201624 